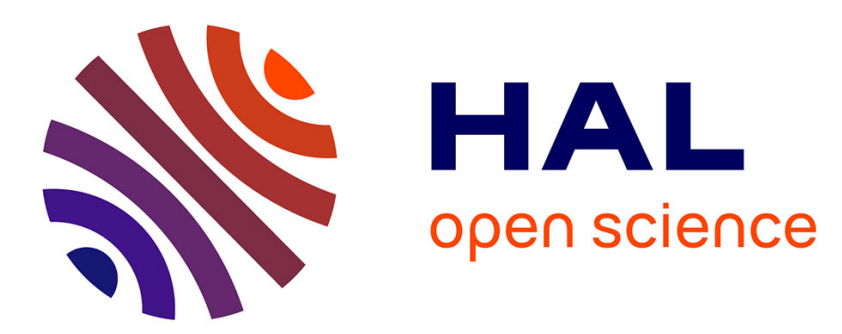

\title{
Motion of solitons of the complex Ginzburg-Landau equation: The effect of an external frequency-shifted source
}

Hervé Leblond, Alioune Niang, Foued Amrani, Mohamed Salhi, François

Sanchez

\section{To cite this version:}

Hervé Leblond, Alioune Niang, Foued Amrani, Mohamed Salhi, François Sanchez. Motion of solitons of the complex Ginzburg-Landau equation: The effect of an external frequency-shifted source. Physical Review A: Atomic, molecular, and optical physics [1990-2015], 2013, 88 (3), pp.033809. 10.1103/PhysRevA.88.033809 . hal-03204338

\section{HAL Id: hal-03204338 \\ https://univ-angers.hal.science/hal-03204338}

Submitted on 21 Apr 2021

HAL is a multi-disciplinary open access archive for the deposit and dissemination of scientific research documents, whether they are published or not. The documents may come from teaching and research institutions in France or abroad, or from public or private research centers.
L'archive ouverte pluridisciplinaire HAL, est destinée au dépôt et à la diffusion de documents scientifiques de niveau recherche, publiés ou non, émanant des établissements d'enseignement et de recherche français ou étrangers, des laboratoires publics ou privés. 


\title{
Motion of solitons of the complex Ginzburg-Landau equation: The effect of an external frequency-shifted source
}

\author{
H. Leblond, A. Niang, F. Amrani, M. Salhi, and F. Sanchez \\ LUNAM Université, Université d'Angers, Laboratoire de Photonique d'Angers, EA 4464, 2 Boulevard Lavoisier, \\ 49045 Angers Cedex 01, France \\ (Received 9 July 2013; published 4 September 2013)
}

\begin{abstract}
The effect of an external continuous wave (cw) on the motion of solitons of the complex Ginzburg-Landau (CGL) equation is investigated. It is shown that soliton motion, which is prevented by a strong braking due to finite gain bandwitdh, can be induced by the $\mathrm{cw}$. Then the effect of the cw on the collective behavior of a large number of dissipative solitons is considered. Starting from a soliton crystal, we demonstrate that the soliton motion is induced by the cw, so that either the soliton gas, liquid, or crystal can be obtained depending on the intensity and the frequency of the cw.
\end{abstract}

DOI: 10.1103/PhysRevA.88.033809

PACS number(s): 42.65.Tg, 05.45.Yv, 42.55.Wd

\section{INTRODUCTION}

The interaction of a large number of solitons was first considered in the case of passive resonators [1-5], modeled by the nonlinear Schrödinger (NLS) equation, without any gain, but driven by an external source term. In the so-called dissipative case, i.e., in the situation involving gain as a laser, two- and three-soliton interactions have been considered theoretically in the frame of the complex Ginzburg-Landau (CGL) equation [6-10], and experimentally in fiber lasers [11-14]. Further, in passively mode-locked high power fiber lasers, a large number of solitons can coexist in the cavity when operating in the anomalous dispersion regime [15-17], which allowed experimental and numerical studies of the interaction of a large number of solitons. Many different soliton patterns, analogous to the different states of matter, have been reported independently of the exact mode-locking mechanism revealing some universal properties [18-25]: soliton crystal, soliton liquid, and soliton gas. The resulting soliton distributions in a fiber laser are interpreted as a consequence of their interactions which can be repulsive or attractive or both at different scales. Attractive interaction is claimed to be responsible of bound states or soliton crystals [18,24], a repulsive long-distance interaction is responsible of the harmonic mode-locking [26], while in the soliton gas, the solitons move one with respect to the other due to the absence of interaction (or to its excessive weakness).

Most of the theoretical approaches cited above to describe the dissipative soliton interaction are based on the CGL equation, which is also one of the most relevant models for the description of mode-locked fiber lasers. Namely, the well-known master equation by Haus [27] is nothing but the stationary version of CGL; fiber laser models of cubic and cubic-quintic CGL type have been derived from several specific setups [28-31]; further, extensive mathematical studies are devoted to this model [32]. However, all existing interpretations (including the qualitative interpretation of theoretical analysis whose correctness has not to be questioned) are based on a mechanical analogy of soliton motion, which fundamentally rests on the assumption that the solitons, as quasiparticles, move freely in the absence of interaction. Under this assumption, as mentioned above, the formation of bound solitons or soliton crystal correspond to a strong short-range interaction, the liquid to a weaker one, the harmonic mode-locking to long-distance repulsion, and the soliton gas to no interaction. The theoretical study of collective motion is reliant on the question of finding some interaction potential, which describes the interaction. The trouble is that this picture cannot work because, in contrast to the solitons of the NLS equation, the solitons of CGL cannot move freely due to the spectral bandwidth term. We detail below the reasons and meaning of this fact, although it has in principle been known for a long time, and then try to recover the soliton motion.

The experiment can give us a way of solving the problem. Indeed, in many fiber lasers working in harmonic modelocking, a continuous wave (cw) component is present in the optical spectrum [33-36], suggesting that this component strongly influences soliton interaction [37]. Analogous observation has been made in lasers generating soliton pairs [38] and also in large soliton assemblies. Specifically, it has been shown that "soliton rains" can be triggered by the injection of a cw laser [23]. Based on this observation, we recently considered experimentally the influence of an injected external $\mathrm{cw}$ on the collective behavior of a large number of dissipative solitons in a passively mode-locked Er:Yb-doped double-clad fiber laser [39]. We consider in the present paper the effect of a frequency-shifted source term able to model such injection of a cw component on solitons of the CGL equation. We will show that in some sense it allows to restore the soliton motion, but that the picture of the Newtonian mechanics still does not apply to the solitons. Then, considering a soliton assembly, we will see that its organization can be changed from crystal to liquid or gas by the injected cw component.

\section{MOBILITY OF CGL SOLITONS}

The CGL equation reads as

$$
\begin{aligned}
\frac{\partial E}{\partial z}= & \delta E+\left(\beta+i \frac{D}{2}\right) \frac{\partial^{2} E}{\partial t^{2}} \\
& +(\varepsilon+i) E|E|^{2}+(\mu+i v) E|E|^{4}
\end{aligned}
$$

where $E$ is the electric field amplitude, and the real normalized coefficients $\delta, \beta, D= \pm 1, \varepsilon, \mu$, and $\nu$ account for (algebraic) 


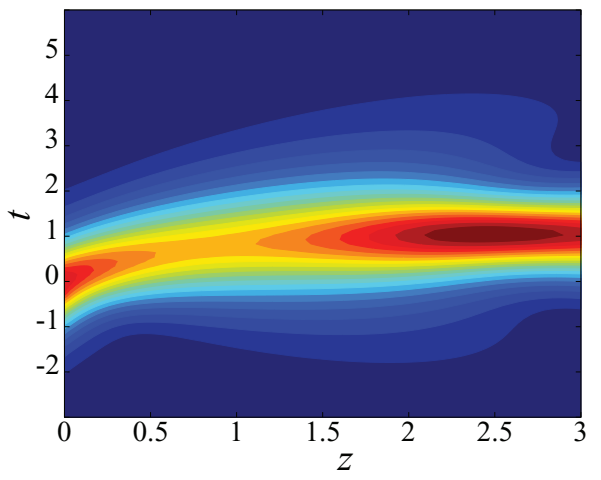

FIG. 1. (Color online) Braking of the soliton for nonzero $\beta$.

net linear gain, spectral gain bandwidth, dispersion, cubic and quintic nonlinear gain, and fourth-order nonlinear index, respectively. When Eq. (1) describes the behavior of a laser cavity, $z$ is the number of round-trips, replaced by a continuous variable by means of a mean-field approximation. As mentioned above, the term $\beta \partial^{2} E / \partial t^{2}$, which accounts for finite spectral bandwidth of the gain, breaks the Galilean invariance of the CGL equation, and prevents any motion of the solitons. More specifically, if $\beta$ is zero, and $E_{0}(z, t)$ is a solution to Eq. (1), then

$$
E=E_{0}(z, t-w z) \exp i\left[D w t-\left(D w^{2} / 2\right) z\right]
$$

is another solution moving at inverse speed $w$.

This is illustrated by numerical resolution of the CGL equation, see Figs. 1 and 2. Equation (1) is solved by means of a standard fourth-order Runge-Kutta algorithm in the Fourier domain, the nonlinear terms being evaluated at each substep by means of inverse and direct fast Fourier transforms. We use an initial data of the form

$$
E(z=0, t)=E_{0}(0, t) e^{i \Delta \omega_{1} t},
$$

where $E_{0}(z, t)$ is a stationary solution of Eq. (1) and $\Delta \omega_{1}$ a frequency shift. When $\beta$ is zero, the frequency shift induces the inverse velocity $w=D \Delta \omega_{1}$, according to Eq. (2). We use the parameters $\delta=-0.01, \beta=0.5, D=1, \varepsilon=0.4, \mu=-0.05$, and $v=0$ (here and throughout the paper, except for Fig. 2 where $\beta$ is replaced with zero). Further, $\Delta \omega_{1}=1.5$ in the data presented in Figs. 1 and 2. Since $\beta$ is not zero, the solution

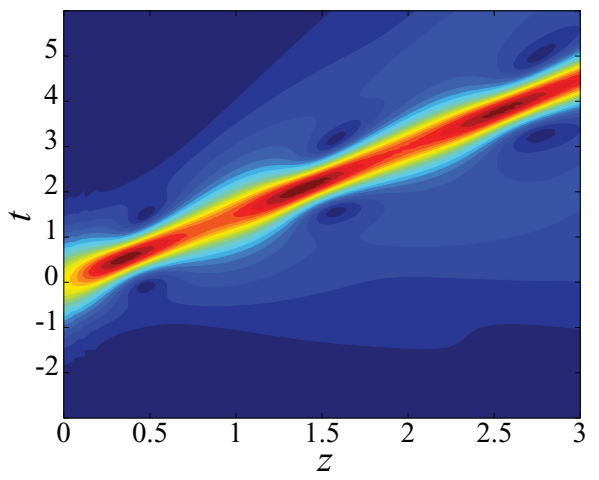

FIG. 2. (Color online) Motion of the soliton for $\beta=0$. given by Eq. (2) does not exist, and numerical solution shows that the soliton with given initial momentum very soon slows down and stops, as can be seen in the example in Fig. 1. The strong braking of the moving soliton due to the limited gain bandwidth term clearly appears. The amplitude and shape of the soliton appreciably change during the braking process; in fact, higher values of $\Delta \omega_{1}$ may lead to the destruction of the soliton. If we switch $\beta$ to zero, using the same parameters and initial data as above, the motion occurs, as shown in Fig. 2, in accordance with the Galilean invariance expressed by Eq. (2). Some oscillations arise due to the fact that the initial data are not exactly a steady state with $\beta=0$ (the steady state exists but is unstable).

To address the question, whether soliton motion can be restored by the injected $\mathrm{cw}$, we consider the equation

$$
\begin{aligned}
\frac{\partial E}{\partial z}= & \delta E+\left(\beta+i \frac{D}{2}\right) \frac{\partial^{2} E}{\partial t^{2}}+(\varepsilon+i) E|E|^{2} \\
& +(\mu+i \nu) E|E|^{4}+A \exp \left(-i \Delta \omega_{0} t\right)
\end{aligned}
$$

which is Eq. (1) with an additional source term. $A$ accounts for the amplitude of the injected $\mathrm{cw}$ and $\Delta \omega_{0}$ is the frequency shift. A $z$-dependent phase factor could be introduced in the frequency-shifted source term. However, since there is no reason that the injecting laser is synchronized with the injected one, this factor, which represents the phase mismatch between both cavities, can be whatever. In the absence of any reasonably founded evaluation of such a factor, we prefer to omit it. Equation (4) is solved by the same method as Eq. (1) above, with the same parameters.

We perform a first set of computations, starting from initial data of the form (3) where $E_{0}$ is a one-soliton solution to the CGL equation (1) [i.e., Eq. (4) with $A=0$ ], and varying $A$, $\Delta \omega_{0}$, and $\Delta \omega_{1}$. It is seen that the pulse acquires some velocity, which depends on $A$ and $\Delta \omega_{0}$, but not on $\Delta \omega_{1}$. In other words, the soliton moves, but the speed is entirely determined by the injected cw signal: the Galilean invariance is not restored at all. We then set $\Delta \omega_{1}=0$, and evaluate the soliton speed (relative to the linear velocity, obviously), or more exactly, the inverse velocity $w=\Delta t / \Delta z$, as a function of the amplitude $A$ and the detuning $\Delta \omega_{0}$ of the cw component.

The results are presented in Fig. 3. At small detuning, and above some amplitude threshold (slightly larger than $A=0.001$ at $\left.\Delta \omega_{0}=0\right)$, the cw component destabilizes the single soliton, and one further soliton may form, or more. See an example obtained for a relatively low amplitude $A=0.01$ and low detuning $\Delta v_{0}=0.05$ in Fig. 4. The rate at which the instability develops increases as the amplitude $A$ increases and as the detuning $\Delta \omega_{0}=2 \pi \Delta v_{0}$ decreases. The number of solitons generated at the end of the process increases in the same conditions, until they fill the computation box. An example of instability, starting from a two-soliton input (a linear superposition of two well-separated fundamental solitons), for $A=0.15$ and $v_{0}=0.12$ is shown in Fig. 5 . Note that, at such a relatively high amplitude of injected cw, before the permanent regime is reached, a cw wave with rather important amplitude forms in parts of the cavity [the dark gray (red) zones about $z=20$ in Fig. 5], however, it is not stable and a series of pulses arises from it. In a laser cavity, the instability 


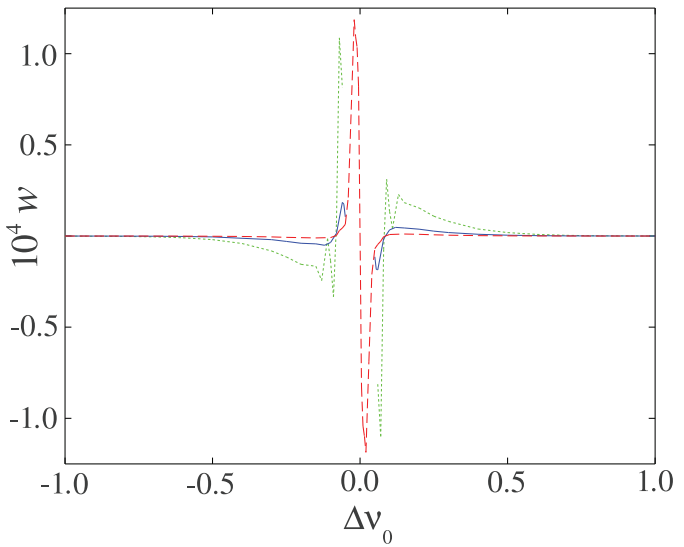

FIG. 3. (Color online) Soliton inverse velocity $w=\Delta t / \Delta z$ against detuning $\Delta v_{0}=\Delta \omega_{0} / 2 \pi$ for a few values of the injected $\mathrm{cw}$ amplitude, namely $A=0.004$ (green dotted line), 0.002 (red dashed line), and 0.001 (blue solid line).

we just described is counterbalanced by the saturation of the gain, which is not taken into account by the CGL model we are considering here. Hence this instability is a property of the mathematical CGL model, which does not correspond to a property of the laser. We limited the computation of velocity to the stability domain of the one soliton. Notice that in this case the velocity $w$ has a very low value, less than $10^{-4}$ (we record them by running the resolution of the propagation equation until $z=20000)$.

\section{CHANGING "STATES OF MATTER" IN A ASSEMBLY OF SOLITONS}

Let us now turn to a large assembly of solitons. We still solve numerically Eq. (4), with the same fixed parameters as above and varying the injected cw amplitude $A$ and frequency shift $\Delta v_{0}=\Delta \omega_{0} / 2 \pi$, but now we use as initial data a soliton crystal solution of Eq. (1) filling the whole computation box, and record the permanent (but not stationary in general) regime which is reached after some transient. This transient corresponds to the stage of development of the instability mentioned in the previous section, however, the soliton crystal may remain stable in the presence of the $\mathrm{cw}$ component with

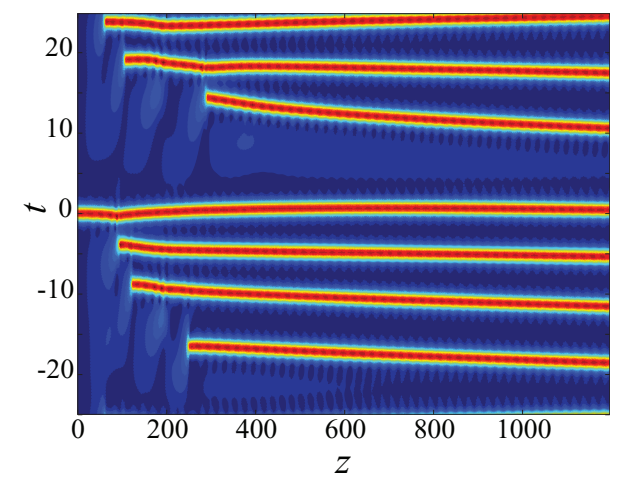

FIG. 4. (Color online) The instability of a single soliton for small cw detuning $\Delta v_{0}=0.05$; the injected cw amplitude is $A=0.01$.

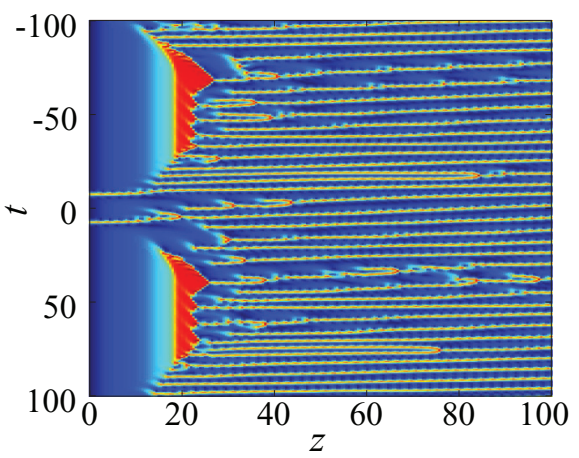

FIG. 5. (Color online) The instability of a two-soliton input for larger cw amplitude is $A=0.15$. The cw detuning is $\Delta v_{0}=0.12$.

amplitude in the considered range ( 0.1 to 2$)$, in contrast with the single soliton. The results do not depend on the sign of $\Delta v_{0}$; depending on its value, the pulse train may behave as a crystal (Fig. 6), a liquid (Fig. 7), or a gas (Fig. 8), which all are computed for the same value of the injected $\mathrm{cw}$ amplitude $(A=1.5)$. Note that this value is quite high with respect to the one considered in the previous section.

The optical spectra computed by means of a fast Fourier transform of the field $E$ for the same three examples are given in Fig. 9, and the autocorrelation trace

$$
\int_{-\infty}^{+\infty}|E(\tau-t) E(\tau)|^{2} d \tau
$$

averaged on ten numerical steps, are shown on Fig. 10.

The various outcomes are shown versus the amplitude and detuning in Fig. 11. The soliton crystal remains if the amplitude is small or the detuning large, a gas is produced in the opposite case. Intermediate patterns form, of liquid or polycrystal type, in between.

Hence, in the frame of the CGL equation, the injection of a cw component allows to control the "states of matter" corresponding to the soliton distribution and also allows, only tuning the frequency of the $\mathrm{cw}$, to change the multisoliton structure, from soliton crystal to soliton gas, via soliton liquid and polycrystal.

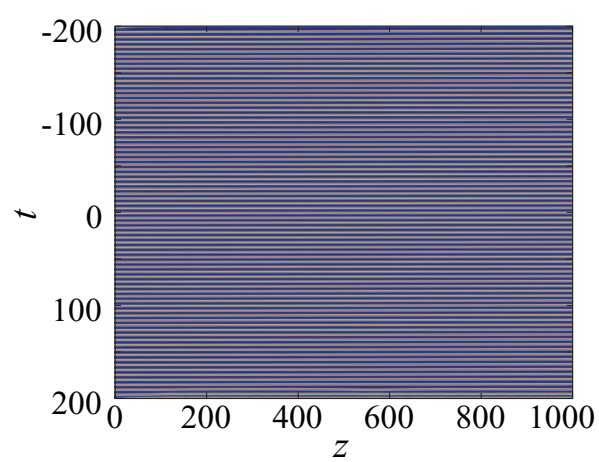

FIG. 6. (Color online) Evolution of the temporal soliton pattern: Soliton crystal. For the value of the detuning $\Delta v_{0}=1.2$. 


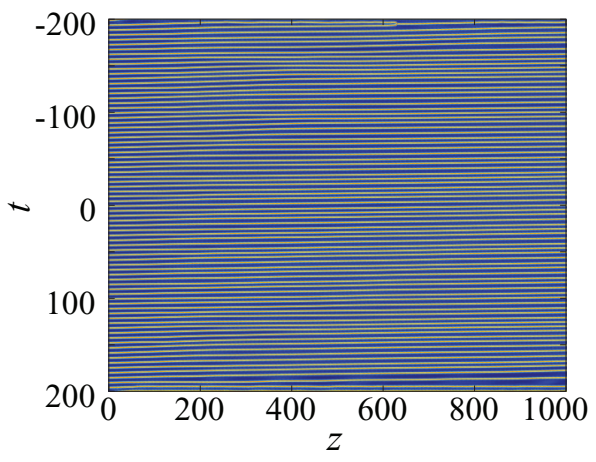

FIG. 7. (Color online) Evolution of the temporal soliton pattern: Soliton liquid. For the value of the detuning $\Delta v_{0}=0.9$.

\section{DISCUSSION}

We started from the observation that the CGL solitons cannot move, which prevents their perpetual motion in a soliton gas, although the latter behavior has been experimentally observed in fiber lasers. The question was whether an injected $\mathrm{cw}$ component was able to put the solitons in motion. We first have shown that, if its amplitude is small, the cw component induces soliton motion. However, the soliton is not really free to move because the velocity depends on the detuning and amplitude of the injected cw component as a single-valued function. It varies strongly with the detuning and amplitudes, suffering high oscillations in absolute value and sign changes, especially for small detunings.

The one-soliton solution to the CGL equation does not remain stable if the amplitude of the injected cw is increased, especially at low detunings: other pulses form, as the amplitude is increased, until they fill the computation box. This instability is not physical since gain saturation occurs in a real laser, but we have to take it into account in the mathematical analysis of CGL.

In correlation with the formation of a great number of solitons under the effect of the cw component, we inspected the influence of the cw component on a large assembly of solitons. We considered a soliton crystal, and show that, varying only the detuning of the cw component, it was possible to transform it into soliton liquid or gas, or to leave it unchanged. Hence the presence of a cw component can induce the apparent "Brownian motion" in a soliton gas. It was shown above that

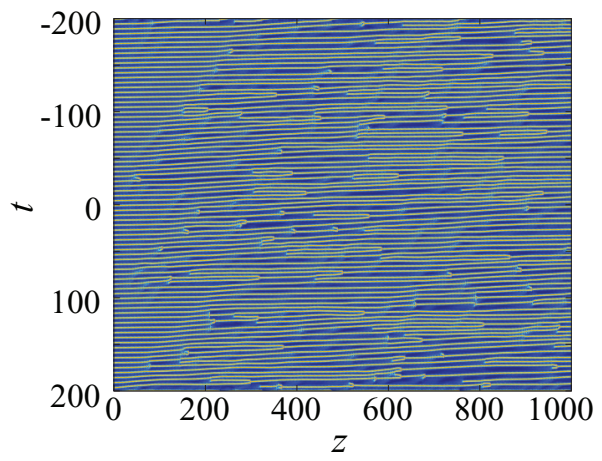

FIG. 8. (Color online) Evolution of the temporal soliton pattern: Soliton gas. For the value of the detuning $\Delta v_{0}=0.8$.

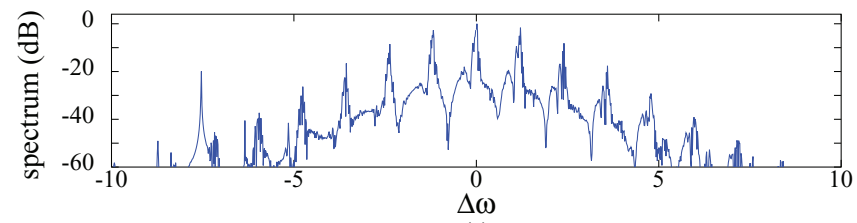

(a)

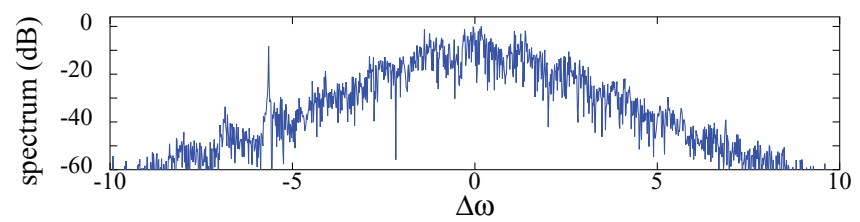

(b)

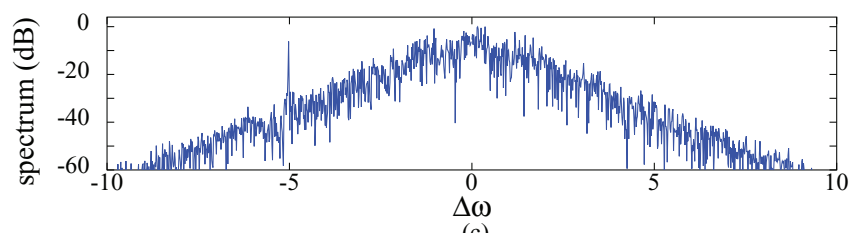

FIG. 9. (Color online) Spectrum for the same three values of detuning as in Figs. 6, 7, and 8. (a) soliton crystal, (b) soliton liquid, and (c) soliton gas.

the velocity induced by the cw component is entirely defined by it. However, at the high amplitudes of injected cw considered in Sec. III, the nonlinear interaction between cw component and pulses is much more complex. In particular, one can expect that the amplitude of the considered radiative wave varies all along the cavity. Since we have seen that a tiny variation of the $\mathrm{cw}$ component in either amplitude or frequency was able to change radically the soliton velocity, the variations of the radiative waves change the soliton in the same way, and the resulting changes are apparently random.

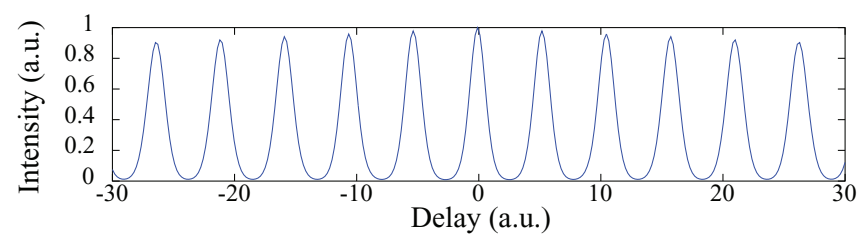

(a)

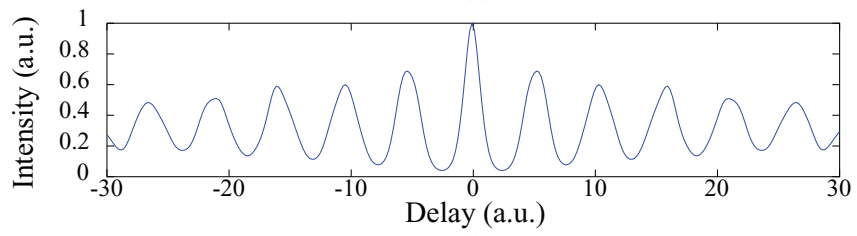

(b)

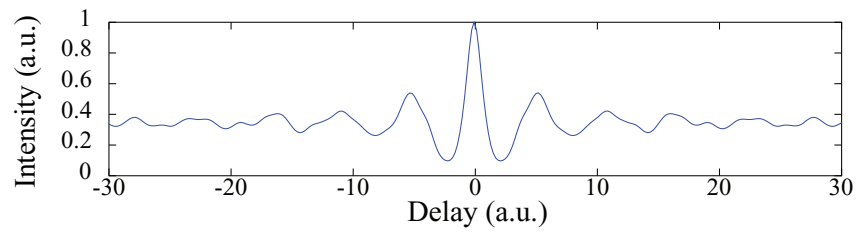

(c)

FIG. 10. (Color online) Autocorrelation trace for the same three values of detuning as in Figs. 6, 7, and 8. (a) soliton crystal, (b) soliton liquid, and (c) soliton gas. 


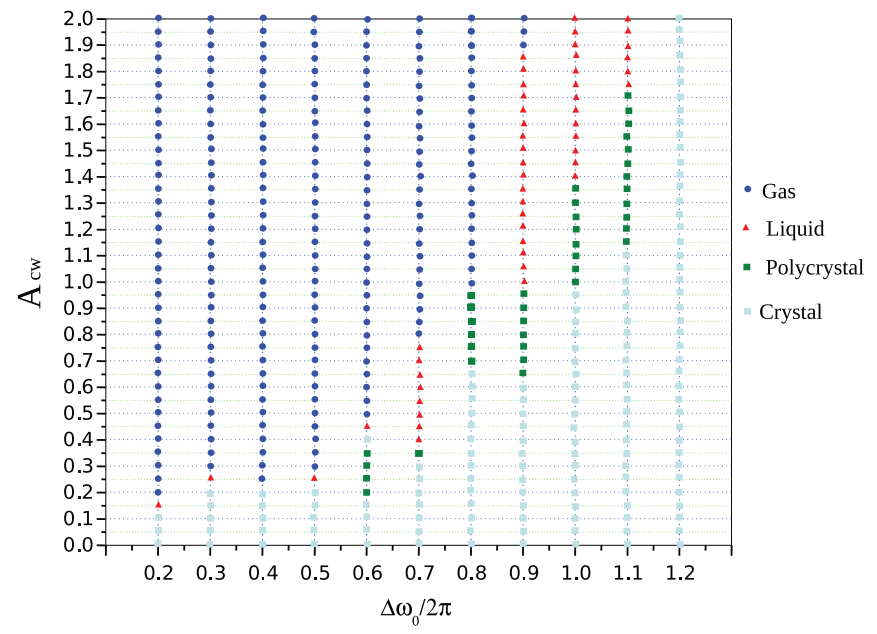

FIG. 11. (Color online) The different regimes versus the detuning $\Delta \omega_{0}$ and the amplitude $A=A_{\mathrm{cw}}$ of the injected cw component.

This is the explanation of the erratic motion in a soliton gas: radiative waves form in a quite erratic way. They induce soliton motion. The relation between the radiative waves and soliton velocity is single-valued but very complicated, so its deterministic character does not prevent the general motion to appear as stochastic. This is a first step towards the theoretical description of the collective behavior of large assemblies of solitons, whose study has been up to now purely descriptive. However, many points remain to be clarified. We still need a simplified model, describing soliton interactions as quasiparticles ones, although we understand that the usual Newtonian picture might be irrelevant here. The better understanding of the motion mechanism will help to the construction of this model. On the other hand, the influence of several further phenomena, which are present in the laser cavity and not accounted for by the CGL equation, especially gain saturation and gain relaxation, remain to be clarified.

\section{ACKNOWLEDGMENT}

We thank the Agence Nationale de la Recherche for supporting this work (Contract No. ANR-2010-BLANC-041701-SOLICRISTAL).
[1] A. Schwache and F. Mitschke, Phys. Rev. E 55, 7720 (1997).

[2] B. A. Malomed, A. Schwache, and F. Mitschke, Fiber Integ. Opt. 17, 267 (1998).

[3] F. Mitschke and A. Schwache, Quantum Semiclass. Opt. 10, 779 (1998).

[4] S. Rutz and F. Mitschke, J. Opt. B: Quantum Semiclass. Opt. 2, 364 (2000).

[5] S. Rutz, T. Körösi, and F. Mitschke, Appl. Phys. B 72, 101 (2001).

[6] B. A. Malomed, Phys. Rev. A 44, 6954 (1991).

[7] V. V. Afanasjev, B. A. Malomed, and P. L. Chu, Phys. Rev. E 56, 6020 (1997).

[8] N. N. Akhmediev, A. Ankiewicz, and J. M. Soto-Crespo, Phys. Rev. Lett. 79, 4047 (1997).

[9] N. N. Akhmediev, A. Ankiewicz, and J. M. Soto-Crespo, J. Opt. Soc. Am. B 15, 515 (1998).

[10] H. Leblond, A. Komarov, M. Salhi, A. Haboucha, and F. Sanchez, J. Opt. A: Pure Appl. Opt. 8, 319 (2006).

[11] A. Komarov, A. Haboucha, K. Komarov, H. Leblond, M. Salhi, and F. Sanchez, in Recent Research Developments in Optics, Vol. 7, Part I, edited by S. G. Pandalai (Research Signpost, Trivandrum, India, 2009), pp. 63-112.

[12] D. Y. Tang, B. Zhao, L. M. Zhao, and H. Y. Tam, Phys. Rev. E 72, 016616 (2005).

[13] Y. D. Gong, P. Shum, T. Hiang, Cheng, Q. Wen, and D. Y. Tang, Opt. Com. 200, 389 (2001).

[14] Ph. Grelu, F. Belhache, F. Gutty, and J. M. Soto-Crespo, Opt. Lett. 27, 966 (2002).

[15] A. Komarov, H. Leblond, and F. Sanchez, Phys. Rev. A 71, 053809 (2005)

[16] X. Liu, Phys. Rev. A 81, 023811 (2010).

[17] D.Y. Tang, L. M. Zhao, B. Zhao, and A. Q. Liu, Phys. Rev. A 72, 043816 (2005).
[18] A. Haboucha, H. Leblond, M. Salhi, A. Komarov, and F. Sanchez, Opt. Lett. 33, 524 (2008).

[19] F. Amrani, A. Haboucha, M. Salhi, H. Leblond, A. Komarov, and F. Sanchez, Appl. Phys. B 99, 107 (2010).

[20] F. Amrani, M. Salhi, H. Leblond, Ph. Grelu, and F. Sanchez, Opt. Lett. 36, 1545 (2011).

[21] A. Zaviyalov, R. Iliew, O. Egorov, and F. Lederer, Appl. Phys. B 104, 513 (2011)

[22] Ph. Grelu and J. M. Soto-Crespo, J. Opt. B: Quantum Semiclass. Opt. 6, S271 (2004).

[23] S. Chouli and Ph. Grelu, Opt. Exp. 17, 11776 (2009).

[24] A. Haboucha, H. Leblond, M. Salhi, A. Komarov, and F. Sanchez, Phys. Rev. A 78, 043806 (2008).

[25] A. Komarov, A. Haboucha, and F. Sanchez, Opt. Lett. 33, 2254 (2008).

[26] A. B. Grudinin and S. Gray, J. Opt. Soc. Am. B. 14, 144 (1997).

[27] H. A. Haus, K. Tamura, L. E. Nelson, and E. P. Ippen, IEEE J. Quantum Electron. 31, 591 (1995).

[28] H. Leblond, M. Salhi, A. Hideur, T. Chartier, M. Brunel, and F. Sanchez, Phys. Rev. A 65, 063811 (2002).

[29] A. Komarov, H. Leblond, and F. Sanchez, Phys. Rev. E 72, 025604(R) (2005).

[30] E. Ding and J. N. Kutz, J. Opt. Soc. Am. B 26, 2290 (2009).

[31] M. Salhi, A. Haboucha, H. Leblond, and F. Sanchez, Phys. Rev. A 77, 033828 (2008)

[32] I. S. Aranson and L. Kramer, Rev. Modern Phys. 74, 99 (2002).

[33] F. Amrani, A. Haboucha, M. Salhi, H. Leblond, A. Komarov, Ph. Grelu, and F. Sanchez, Opt. Lett. 34, 2120 (2009).

[34] G. Sobon, K. Krzempek, P. Kaczmarek, K. M. Abramski, and M. Nikodem, Opt. Com. 284, 4203 (2011).

[35] Z. X. Zhang, L. Zhan, X. X. Yang, S. Y. Luo, and Y. X. Xia, Laser Phys. Lett. 4, 592 (2007). 
[36] F. Amrani, A. Niang, M. Salhi, H. Leblond, and F. Sanchez, Opt. Lett. 36, 4239 (2011).

[37] A. Komarov, K. Komarov, H. Leblond, and F. Sanchez, J. Opt. A: Pure Appl. Opt. 9, 1149 (2007).
[38] J. M. Soto-Crespo, N. Akhmediev, Ph. Grelu, and F. Belhache, Opt. Lett. 28, 1757 (2003).

[39] A. Niang, F. Amrani, M. Salhi, H. Leblond, A. Komarov, and F. Sanchez, Opt. Com. (to be published). 